\title{
Necrópsia: valor diagnóstico
}

\section{Necropsy: diagnostic value}

Márcia Valéria Pitombeira Ferreira ${ }^{1}$. Venulda Helena Santos Mendes ${ }^{2}$. Tainah Braga Camurça ${ }^{3}$. Luanna de Queiroz Lemos ${ }^{3}$. Oálene Gonçalves Silva ${ }^{4}$. Carolina Teixeira Costa ${ }^{3}$. Thamiris Silva de Queiroz .

1 Mestrado em Patologia, Departamento de Patologia da Faculdade de Medicina, Universidade Federal do Ceará, Fortaleza, Ceará, Brasil. 2 Graduação em Medicina, Universidad de Ciencias Médicas de Camaguey, Cuba. Médica do Programa de pós-graduação Lato Sensu em Patologia pelo Hospital Universitário Walter Cantídeo (HUWC), Universidade Federal do Ceará (UFC), Fortaleza, Ceará, Brasil. 3 Acadêmica do Curso de Medicina, Universidade Federal do Ceará (UFC), Fortaleza, Ceará, Brasil. 4 Graduação em Medicina, Universidade Federal do Ceará (UFC), Fortaleza, Ceará, Brasil.

\section{RESUMO}

Introdução: a necrópsia garante a acurácia estatística de mortalidade, esclarece mortes não explicadas, é fonte de aprendizado, de pesquisa e controle de qualidade hospitalar. Objetivos: verificar a frequência de necrópsias no Departamento de Patologia e Medicina Legal da Universidade Federal do Ceará (DPML/UFC), avaliar a eficácia diagnóstica da necrópsia, concordância e discordância entre o diagnóstico clínico e de necrópsia. Metodologia: estudo longitudinal, retrospectivo, descritivo, de levantamentos de dados dos arquivos do DPML/UFC de 1959 a 2014 para frequência anual e total, e de 2010 a 2014 para analisar os diagnósticos clínico e de necrópsia. Resultados: de 1959 a 2014 foram 7186 necrópsias. Na década de 80 foram mais de 200 anuais, e a partir dos anos 90 houve declínio. De 2010 a 2014 foram cerca de 50/ano. O diagnóstico clínico foi discordante com o da necrópsia em $23 \%$ dos casos adultos e jovens. Nos natimortos e óbitos infantis o diagnóstico clínico foi discordante em $15 \%$ com o diagnóstico da necrópsia. A necrópsia foi conclusiva em $86 \%$ de adultos e jovens e $78 \%$ dos fetos e óbitos infantis. Conclusão: houve redução das necrópsias, a precisão da necrópsia foi elevada e discordâncias foram compatíveis com a literatura.

Palavras-chave: Autópsia. Causa de morte. Diagnóstico clínico. Erros de diagnóstico. Hospitais universitários. Necrópsia. Mortalidade hospitalar.

\section{ABSTRACT}

Introduction: Necropsy guaranties the accuracy of mortality statistics, clarifies unexplained deaths and is a source for learning, research and control of hospital quality. Objectives: Check the autopsies frequencies in the Department of Pathology and Legal Medicine of the Universidade Federal do Ceará (DPLM/UFC), evaluate the accuracy of diagnoses, determine the diagnostic agreement/discrepancy between clinical and autopsy diagnoses. Methodology: longitudinal study, retrospective, descriptive, of data surveys of the files on the DPLM/UFC from 1959 to 2014 to consider annual and total frequencies, and from 2010 to 2014 in order to analyze clinical and autopsy diagnoses. Results: From 1959 to 2014 there were 7186 autopsies. During the 80s there were over than 200/year. From the 1990s on there was a decline. From 2010-2014 the average lowers down to 50/year. Clinical diagnoses were inconsistent with the autopsy in $23 \%$ of adults and young cases. In infant deaths and stillbirths the clinical diagnoses was $15 \%$ discordant with the diagnoses of necropsy. Autopsies were conclusive in $86 \%$ of the adults and young people and in $78 \%$ of the fetuses and infant deaths. Conclusion: There was a large reduction in the number of the autopsies, the necropsies were diagnosed with high precision and disagreements were compatible with the literature.

Keywords: Autopsy. Cause of death. Clinical diagnosis. Diagnostic errors. Hospital mortality. Necropsy. Hospitals, University.

Autor correspondente: Márcia Valéria Pitombeira Ferreira, Rua Silva Jathay, 1350, Meireles, Fortaleza, Ceará. CEP: 60165-070.Telefone: +55 85 3242-1373/98103-5768. E-mail: mvpitombeira@yahoo.com.br

Conflito de interesses: Não há qualquer conflito de interesses por parte de qualquer um dos autores.

Recebido em: 20 Jul 2017; Revisado em: 09 Set 2017; Aceito em: 21 Set 2017. 


\section{INTRODUÇÃO}

O procedimento de necrópsia garante a acurácia dos atestados de óbito e das estatísticas de mortalidade, esclarece causa de morte não explicada clinicamente, também é uma fonte de aprendizado e de treinamento técnico, além de fonte de pesquisa e de controle de qualidade dos serviços hospitalares. ${ }^{1}$ Importante enfatizar que o residente em anatomia patológica deve realizar necrópsias como parte de seu treinamento, outras residências médicas tem estágios obrigatórios e sessões de integração anatomoclínica no serviço de patologia, como parte da carga-horária. ${ }^{2}$ A literatura relata que há uma redução do número de necrópsias mundialmente, apesar de reconhecida como instrumento padrão-ouro para o diagnóstico e a aferição da qualidade da assistência médica., ${ }^{2,3}$ Nos Estados Unidos, a redução em 2002 atingiu valores de $5 \% \cdot{ }^{4} \mathrm{~A}$ taxa de realização de necrópsia na Austrália reduziu de $21 \%$ no começo dos anos 90 para $12 \%$ em 2003..$^{5}$ No Brasil tem sido observado diminuição progressiva no número de necrópsias hospitalares na maioria das instituições de ensino. ${ }^{2,3} \mathrm{O}$ declínio no número de exames post-mortem não significa necessariamente que houve uma melhora na precisão dos diagnósticos clínicos, ou que os avanços nos métodos diagnósticos substituíram esta prática. A incidência de erros detectados pelas necrópsias permanece elevada, particularmente em se tratando de fetos, neonatos, idosos, imunodeficientes e pacientes em estado crítico. ${ }^{6}$ As taxas de discrepâncias entre os diagnósticos clínicos e patológicos variam de 6 a 45\%, enfatizando a importância desta prática nas instituições hospitalares. ${ }^{7,8}$

A redução do número de necrópsias foi observada no Departamento de Patologia e Medicina Legal (DPML) da Universidade Federal do Ceará(UFC), porém nunca quantificada.

Este trabalho objetivou determinar a frequência anual e total das necrópsias no período de 1959, início de funcionamento do serviço, até 2014 e no período de 2010 a 2014, observar a relação entre o número de óbitos hospitalares e a realização de necrópsia, a concordância e discrepância entre o diagnóstico clínico e patológico, bem como determinar a importância da necrópsia como ferramenta precisa de diagnóstico e também levar à reflexão sobre as consequências da redução de necrópsia hospitalar.

\section{MATERIAL E MÉTODOS}

Este trabalho é um estudo longitudinal, retrospectivo, descritivo, de levantamentos de dados dos arquivos do Serviço de Necrópsia do DPML/UFC. Fizeram parte do estudo todos os casos enviados à necrópsia provenientes do Complexo dos Hospitais Universitários da Universidade Federal do Ceará (HUs/UFC), Hospital São José (HSJ) e algum outro hospital que eventualmente solicitou este exame. Os dados levantados foram para quantificar a frequência anual e total das necrópsias de 1959 a 2014 e no período de 2010 a 2014, para observar a relação entre o número de óbitos hospitalares e a realização de necrópsia, analisar a concordância e discrepância entre os diagnósticos clínicos e de necrópsia e a necrópsia como ferramenta diagnóstica. Foram avaliadas as necrópsias realizadas em adultos e jovens e as necrópsias realizadas em natimortos e óbitos infantis. O projeto foi aprovado pelo comitê de ética em pesquisa do Hospital Universitário Walter Cantídio (HUWC), parecer $\mathrm{N}^{\circ}$ 1.198.853. Para cálculos e gráficos foram utilizados Excel 2013 e software R 3.3.1.

\section{RESULTADOS}

De 1959 a 2014 foram realizadas 7186 necrópsias. O Gráfico 1 mostra redução do número de necrópsias ao longo de 55 anos no DPML/UFC. Nos períodos áureos ocorreram mais de 200 necrópsias/ano, chegando a 309 em 1981. De 2010 a 2014 o número de casos se manteve em torno de 50 por ano.

De 2010 a 2014 foram 238 necrópsias. Destas, 134 corresponderam a natimortos ébitos infantis, $98 \%$ provenientes da Maternidade Escola Assis Chateaubriand (MEAC), 90\% natimortos e $51 \%$ do sexo masculino. Os óbitos de adultos e jovens foram 104 casos, 69\% do HUWC, 24\% HSJ, 3\% MEAC e $4 \%$ de outros hospitais, $57 \%$ foram do sexo masculino, estes com a média de idade de 45 anos, e 47 anos para as mulheres. De 2010 a 2014 a distribuição de casos de necrópsia/ano foi de $27,25,20,20$ e 12 relativos a adultos e jovens e natimortos e óbitos infantis de 23, 25, 33, 29 e 24. Os Gráficos 2 e 3 mostram o número de óbitos dos HUs/UFC no período de 2010 a 2014 relacionando com as necrópsias realizadas, revelando a pequena porcentagem de casos examinados.

Gráfico 1. Distribuição das necrópsias realizadas no DPML/UFC de 1959 a 2014.

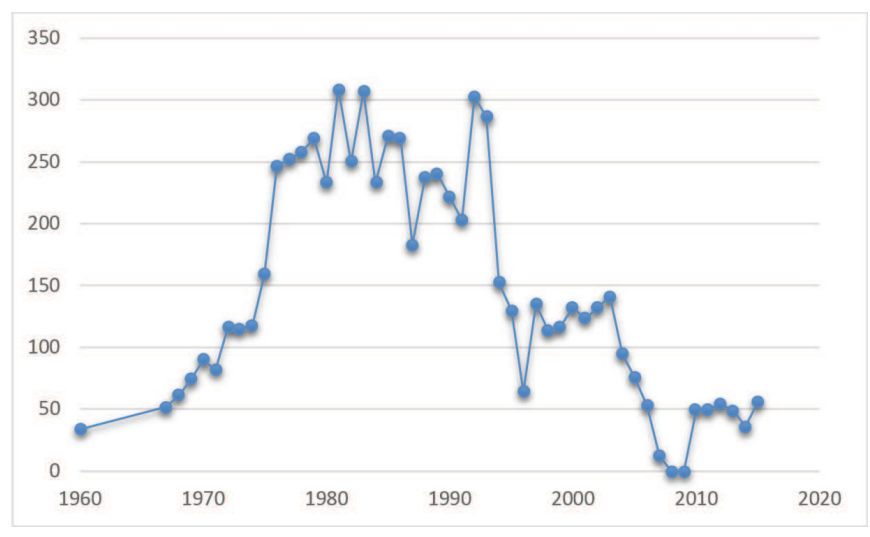

Gráfico 2. Óbitos do complexo dos HUs/UFC e necrópsias realizadas no DPML/UFC de 2010 a 2014.

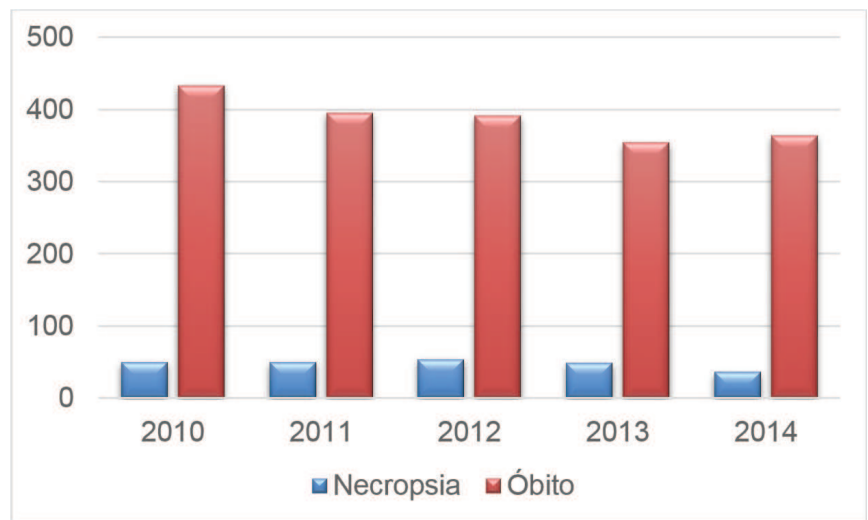


Gráfico 3. Porcentagem de necrópsias nos óbitos do Complexo dos HUs/UFC realizadas no DPML/UFC de 2010 a 2014.

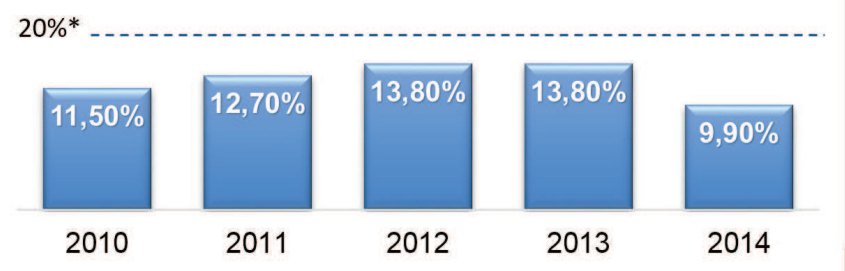

A Tabela 1 evidencia que dos 104 pacientes adultos e jovens o diagnóstico clínico foi discrepante com o diagnóstico de necrópsia em 23\%. Em 77\% dos casos houve algum tipo de concordância entre o diagnóstico clínico com o diagnóstico de necrópsia. A concordância foi total em $46 \%$ dos casos na doença de base, complicações e causa de morte. A concordância parcial entre necrópsia e diagnóstico clínico de $31 \%$ foi devida ao diagnóstico da doença de base do paciente estar de acordo com a doença de base diagnosticada à necrópsia. As discordâncias entre o diagnóstico clínico e de necrópsia foram relativas à falta de definição de complicações e da causa da morte destes pacientes pelo diagnóstico clínico.

Tabela 1. Concordância e discordância entre diagnóstico clínico e diagnóstico de necrópsia em 104 pacientes adultos e jovens e 84 natimortos e óbitos infantis no DPML/UFC de 2010 - 2014.

\begin{tabular}{llll}
\hline \multicolumn{3}{l}{ Concordância } & Discordância \\
\hline Indivíduos & Total & Parcial & \\
& & & \\
Adultos e jovens & $48(46 \%)$ & $32(31 \%)$ & 24(23\%) \\
$\begin{array}{l}\text { Natimortos e } \\
\text { Óbitos infantis }\end{array}$ & $53(63 \%)$ & $18(21 \%)$ & $13(15 \%)$ \\
\hline
\end{tabular}

A Tabela 2 apresenta a eficácia da necrópsia na definição diagnóstica dos casos. O exame de necrópsia com a análise macro e microscópica foi conclusiva na causa de morte, nas complicações e na etiologia em 86\% dos 104 casos de adultos e jovens. Nos 15 casos restantes (14\%) a necrópsia diagnosticou complicações e causa de morte, mas apenas com o estudo macro e microscópico usual não definiu a etiologia. Ora as colorações histoquímicas não evidenciaram micro-organismos ou substâncias anormais para definir o diagnóstico etiológico, ora a doença de base não foi evidenciada à necrópsia, ora a definição da etiologia dependia de resultados de sorologia ou de imunohistoquímica.
Tabela 2. Diagnóstico da necrópsia em 104 pacientes adultos e jovens e 134 natimortos e óbitos infantis no DPML/UFC de 2010 - 2014.

\begin{tabular}{llll}
\hline Indivíduos & $\begin{array}{l}\text { Conclusiva } \\
\text { Total }\end{array}$ & $\begin{array}{l}\text { Conclusiva } \\
\text { Parcial }\end{array}$ & $\begin{array}{l}\text { Nenhuma } \\
\text { Conclusão }\end{array}$ \\
\hline Adultos e jovens & $89(86 \%)$ & $15(14 \%)$ & $0(0 \%)$ \\
& & & \\
$\begin{array}{l}\text { Natimorto e } \\
\text { Óbitos infantis }\end{array}$ & $104(78 \%)$ & $30(22 \%)$ & $0(0 \%)$ \\
\hline
\end{tabular}

Dos 134 casos de natimortos e óbitos e infantis, 84 (63\%) traziam as informações clínicas diagnósticas ou necessárias para o diagnóstico nas requisições de necrópsias, e em 50 (37\%) casos estas informações eram incompletas ou totalmente ausentes, insuficientes para a conclusão diagnóstica à necrópsia. Na Tabela 1 observa-se que houve concordância total com a clínica em $63 \%$ dos 84 casos que continham as informações clínicas, concordância parcial em $21 \%$ e totalmente discordante em 15\%. A Tabela 2 mostra que dos 134 casos de natimortos e óbitos infantis, a necrópsia foi conclusiva em 78\%. Em 30 casos $(22 \%)$ a necrópsia não definiu a etiologia. Nestes casos, o diagnóstico foi apenas de anóxia intraútero e fetos macerados, sem possibilidade de correlação clínico-patológica em decorrência de informação clínica insuficiente ou ausente.

\section{DISCUSSÃO}

O presente estudo constatou a redução importante no número de necrópsias realizadas no DPML/UFC ao longo de 55 anos e que se tornou crítica nas últimas décadas. Ficou também evidenciada na amostragem de 2010 a 2014, a baixa porcentagem de realização de necrópsias nos óbitos hospitalares dos HUs/UFC. Esta realidade não é exclusiva do DPML/UFC e dos HUs/UFC. Recente publicação mostra que no Reino Unido o declínio em autópsia hospitalar tem continuado durante os últimos anos. A taxa em 2013 no Reino Unido foi de $0,69 \%$ de mortes do hospital. Autópsia hospitalar está extinta em $23 \%$ de todo o Reino Unido e está ameaçada de extinção no restante. ${ }^{9}$ No entanto, esta mesma publicação ressalta a importância de mais pesquisas sobre a segurança do paciente, questões de auditoria, investigação, saúde pública e ensino com a redução das necrópsias hospitalares. ${ }^{9}$ A literatura aponta causas múltiplas e complexas para a redução do número de necrópsias no mundo: aspectos religiosos, familiares, mudança na relação entre os médicos, paciente e seus familiares, avanços na tecnologia diagnóstica, a ideia de que agora os diagnósticos podem ser feitos em vida, receio de processos judiciais contra médicos, falta de conhecimento da população sobre a importância da necrópsia, além da necrópsia ser um procedimento caro. Apesar de todas estas causas, o estudo pós-morte permanece útil e eficaz no diagnóstico das doenças, ${ }^{8}$ como evidenciado no presente trabalho, em que a necrópsia foi conclusiva nos diagnósticos em $86 \%$ dos casos dos adultos e jovens. Neste grupo, os casos em que a necrópsia não esclareceu em sua totalidade deveram-se à necessidade de metodologias especiais para a definição etiológica. 
A eficácia diagnóstica foi menor no grupo dos natimortos e óbitos infantis, ficando em $78 \%$. O diagnóstico inconclusivo nas necrópsias deste grupo foi devido à ausência ou pouca informação clínica materno-fetal para integração clínico-patológica necessária ao diagnóstico, principalmente pelo fato de que $90 \%$ dos casos eram natimortos com graus variados de maceração. Esta falta de informação pode estar relacionada, em parte, ao fato de gestantes chegarem em trabalho de parto na emergência da instituição, sem haver realizado pré-natal na mesma.

É imperativo o preenchimento o mais completo possível das requisições de qualquer caso encaminhado à necrópsia, pois todo laudo anatomopatológico é parte integrante do prontuário médico e requer informações precisas.

A discordância diagnóstica entre clínica e patologia em cada grupo foi de acordo com a literatura. ${ }^{3,10}$ Neste estudo constatou-se que a taxa de discordância entre diagnósticos clínicos e post mortem nas amostras de adultos e jovens do período estudado foi de $23 \%$, e nos natimortos e óbitos infantis de $22 \%$. Há na literatura uma variação na taxa de discordância entre diagnóstico clínico e diagnóstico de necrópsia de $6 \%$ e 40\%. ${ }^{11}$ As taxas mais comuns de discordâncias relatadas variam de 20 a 30\%. Superiores a 40\% são alerta de possíveis deficiências ou insuficiências em algum ponto da assistência hospitalar e menores que $10 \%$ pode estar relacionado a inconformidades nos criterios utilizados. Quando em um hospital geral ou clínico os resultados forem menor de $10 \%$ ou maior que $40 \%$ recomenda-se a revisão da metodologia usada para garantir que a avaliação seja fiel à realidade. ${ }^{12}$

É importante investigar os motivos de declínio na realização de necrópsia hospitalar e estabelecer estratégias de incentivo ao seu retorno. Quantos casos um hospital deve solicitar para exame de necrópsia dentro da quantidade de óbito hospitalar? Quais casos devem ser selecionados para a necrópsia? Quais casos possivelmente levarão a discordâncias diagnósticas? Apesar de não haver consenso, há recomendação na literatura de uma taxa mínima de autopsias de $20 \%$ para hospitais de ensino. ${ }^{13}$ Neste trabalho ficou evidente não só a redução acentuada do número de necrópsias ao longo dos 55 anos, como também nas últimas décadas. A relação entre o numero de óbitos dos HUs/UFC e a realização de necrópsias avaliada em 5 anos teve como maior taxa 13,8\%, nos anos de 2012 e 2013, e a menor de 9,9\% em 2014, ficando longe da recomendada na literatura. Esta taxa foi calculada com a unificação do número de óbitos dos dois HUs/UFC. Considerando que a redução em

\section{REFERÊNCIAS}

1. Fares AF, Cury PM, Lobo SM. Discrepâncias clínico-patológicas em pacientes graves com difícil diagnóstico pre-mortem. Rev Bras Ter Intensiva. 2011;23(4):442-7.

2. Rodrigues FR, Lopes VG, Lopez CL, Soares PJ Filho, Silva RC, Silva LE, et al. O decréscimo vertiginoso das autópsias em um hospital universitário do Brasil nos últimos 20 anos. J Bras Patol Med Lab. 2011;47(4):445-50. número de necrópsias vem sendo maior no grupo de adultos e jovens, o percentual de necrópsias neste grupo seria ainda menor se analisado separadamente os HUs/UFC.

Mesmo com os avanços da medicina nos últimos 30 anos, a taxa de morte não explicada permaneceu entre $22 \%$ e $33 \%{ }^{2}{ }^{2}$ E de se supor que esta informação também deva ser levada em conta na definição da taxa mínima de necrópsias dos óbitos em hospitais de ensino.

Esta publicação pretende sensibilizar a respeito do declínio das necrópsias hospitalares e levar à reflexão sobre as suas consequências. O declínio tem também uma implicação direta no ensino da graduação e nas residências médicas, contribuindo para geração de uma cultura de não solicitação de necrópsias quando os médicos estiverem na sua atuação profissional, criando uma situação favorável para a perpetuação da redução do número das necrópsias.

Propiciar a continuidade da utilização das informações oferecidas pelas necrópsias constitui medida vital no aprimoramento dos serviços médicos, do ensino e da pesquisa nas instituições hospitalares, notadamente as de ensino, ${ }^{3}$ principalmente em países com deficiências na assistência à saúde.

A literatura avalia que o uso contínuo da autópsia é justificado, mas a evidência de diminuição nos principais erros de diagnóstico ou um aumento na acuidade da tecnologia de diagnóstico sugere uma necessidade de um número menor de necrópsias que no passado. ${ }^{14}$ Entretanto, deve permanecer o foco da busca do conhecimento e expressar o respeito pelo corpo da pessoa falecida e dos familiares. ${ }^{15}$ Médicos e os parentes dos falecidos têm interesses diferentes, e uma visão diferente sobre a necrópsia, o que sugere a necessidade de mais atenção ao consentimento informado para este exame. Esta ação deve ter prioridade sobre os esforços para aumentar a taxa de necrópsia, sobretudo, melhorando a conscientização e a habilidade dos profissionais em obter o consentimento esclarecido para a necrópsia. ${ }^{15}$

Em conclusão, este trabalho enfatizou a importância da necrópsia como uma ferramenta médica de diagnóstico preciso, que as discordâncias entre o diagnóstico clínico e de necrópsia estão de acordo com as da literatura e que há um declínio acentuado no número de necrópsias no DPML/UFC, reflexo da baixa taxa de necrópsias nos óbitos dos HUs/UFC, o que tem sido visto mundialmente.

3. Moreira DR, Lana AM, Godoy P. Estudo sobre a contribuição da autopsia como método diagnóstico. J Bras Patol Med Lab. 2009;45(3):239-45.

4. Burton EC. The autopsy: a professional responsibility in assuring quality of care. Am J Med Qual. 2002;17(2):56-60.

5. Royal College of Pathologists of Australasia Autopsy Working 
Party. The decline of the hospital autopsy: a safety and quality issue for healthcare in Australia. Med J Aust. 2004;180(6):281-5.

6. Rocha LO. Necrópsia e educação médica. Rev Med Minas Gerais. 2014;24(1): 106-13.

7. Bürgesser MV, Camps D, Calafat P, Diller A. Discrepâncias entre diagnósticos clínicos y hallazgos de autopsia. Medicina (B. Aires). 2011;71(2):135-8.

8. Roulson J, Benbow EW, Hasleton PS. Discrepancies between clinical and autopsy diagnosis and the value of post mortem histology; a meta-analysis and review. Histopathology. 2005;47(6):551-9.

9. Turnbull A, Osborn M, Nicholas N. Hospital autopsy: endangered or extinct? J Clin Pathol. 2015;68(8):601-4.

10. Costache M, Cirstoiu M, Contolenco A, Lazaroiu AM, George S, Sajin M, et al. A comparison between clinical diagnosis of death and autopsy diagnosis. A retrospective study of 131 newborns, stillborns and aborted fetuses. Maedica (Buchar). 2014;9(2):183-8.
11. Castro AG, Llorca J, Fernández-Miret B, Cañas BS, Minãmbres E. Discrepancias entre el diagnóstico clínico y el diagnóstico anatomopatológico em la mortalidad temprana del transplante pulmonar. Med Intensiva. 2009;33(9):424-30.

12. Mendonça AJ, Álvarez SR, Borrajero MI. Discrepancias diagnósticas en las causas de muerte identificadas por autopsias. Cuba 1994-2003. Primera parte. Patol Rev Latinoam. 2008;46(2):8595.

13. Cury MR, Chiaravalotti F Neto, Zanetta DM. Avaliação de concordância entre diagnósticos clínicos e relatórios finais de autópsias em hospital universitário. Arq Cienc Saude. 2005;12(1):37.

14. Shojania KG, Burton EC, McDonald KM, Goldman L. Changes in rates of autopsy-detected diagnostic errors over time: a systematic review. JAMA. 2003;289(21):2849-56.

15. Stempsey WE. The penetrating gaze and the decline of the autopsy. AMA J Ethics. 2016;18(8):833-8.

\section{Como citar:}

Ferreira MV, Mendes VH, Camurça TB, Lemos LQ, Silva OG, Costa CT, et al. Necrópsia: valor diagnóstico. Rev Med UFC. 2018 abr-jun;58(2):31-35. 